\title{
COVID-19 Vaccine Uptake Among College Students at a Midwest University
}

\author{
Amy J. Wotring ${ }^{1} \mathbb{D} \cdot$ Matthew Hutchins ${ }^{1} \cdot$ Maureen K. Johnson ${ }^{1} \cdot$ Shiaw-Fen Ferng ${ }^{1} \cdot$ Cassondra Strawser $^{1}$. \\ Heather Pfrank ${ }^{1} \cdot$ Matthew Warner $^{1} \cdot$ Linda Behrendt $^{1}$
}

Accepted: 10 November 2021 / Published online: 20 November 2021

(c) The Author(s), under exclusive licence to Springer Science+Business Media, LLC, part of Springer Nature 2021

\begin{abstract}
COVID-19 is responsible for over 750,000 Americans deaths. Despite efforts to vaccinate, many college students are hesitant. Hesitancy has hindered efforts to achieve high COVID-19 vaccination rates. Students at a Midwest university completed an online survey. Questions included COVID-19 vaccination intent and behavior. Over 1600 students participated. Half reported being vaccinated. Of those not vaccinated, $49 \%$ did not intend to get vaccinated, and $22 \%$ were undecided. Reasons for hesitancy included not trusting the vaccine was fully tested (85\%), fear of potential side effects (78\%), not trusting the vaccine is safe (72\%), not trusting the US government (61\%), and having read negative reports from the media about the vaccine $(60 \%)$. Additionally, students were concerned the vaccine was not safe $\left(B=-0.902 ; 95 \% X^{2}=11.68 ; p=0.001\right)$ and that it could not protect them from COVID-19 $\left(B=-0.834 ; X^{2}=12.43 ; p<0.001\right)$. Students' religious beliefs $\left(B=-1.245 ; X^{2}=4.61\right.$; $p=0.032)$ and having unvaccinated family members $\left(B=-0672 ; X^{2}=11.50 ; p=0.001\right)$ also contributed to intention not to receive the vaccine. Unexpectedly, students reporting fear of potential side effects $\left(B=1.26 ; X^{2}=12.86 ; p<0.001\right)$ were significantly more likely to indicate intention to get vaccinated than students who did not report this fear. College students may be more hesitant to receive the vaccination than others. Targeted messaging addressing concerns/fears may be a way to influence those who are hesitant to receive the COVID-19 vaccination.
\end{abstract}

Keywords COVID-19 $\cdot$ College students $\cdot$ Vaccine hesitancy $\cdot$ Vaccination

\section{Introduction}

According to the Centers for Disease Control and Prevention (CDC) and Johns Hopkins, COVID-19 is responsible for more than 750,000 Americans deaths (Johns Hopkins, 2021) [4]. Economic costs in the United States are estimated at over $\$ 16$ trillion when accounting for health costs and loss of gross domestic product [6]. Despite huge efforts made to achieve successful COVID-19 vaccines, many Americans are not yet vaccinated. Survey data from 2020 suggested $60-70 \%$ of United States adults intended to get vaccinated [2]. Public health experts agree that a $70 \%$ vaccination rate would not meet the threshold needed to achieve herd immunity [10]. Current data show that only $57.5 \%$ of eligible

Amy J. Wotring

Amy.wotring@indstate.edu

1 Department of Applied Health Sciences, College of Health and Human Services, Indiana State University, Terre Haute, USA
United States adults has been vaccinated [4]. A major hindrance has been labelled vaccine hesitancy [9]. Vaccine hesitancy is complex and refers to a delay in acceptance or refusal of vaccination despite the availability of vaccination services. A continuum exists between those who accept vaccines and those who completely refuse vaccination. Hesitancy can be attributed to factors like complacency, convenience, and confidence. One population where hesitancy may be high is college students. Studies have shown that most college students (90.5\%) perceive the COVID-19 vaccination as important, that everyone eligible should be vaccinated $(92.6 \%)$, and most $(67.9 \%)$ believe that the way to overcome the COVID-19 pandemic is through mass vaccination [14].

On the other hand, numerous studies find that nearly half of students report they would not get vaccinated or are not sure if they would seek vaccination [17]. Saied et al. found that only $6 \%$ of students said they would definitely accept the vaccine. One reason for this is that surveys reveal a significant rate of distrust against vaccines and negative attitudes 
towards the COVID-19 vaccination [18]. It is obvious that attitude toward vaccination affects vaccination decisions. It is also apparent that attitudes toward vaccination can change over the course of a public health crisis. Fridman, Gershon, and Gneezy [8] report results from a six-month study of United States residents. Surprisingly, they found a decrease in intention of getting a COVID-19 vaccine and in general decline in attitudes and intentions of getting the influenza vaccine over the course of their longitudinal surveys. Those with less favorable attitudes toward a COVID-19 vaccination perceived the virus to be less threatening. Political affiliation was also found to influence attitudes toward vaccination. Republicans were more likely to have negative attitudes towards the vaccine. Other barriers to COVID-19 vaccination may be deficient data regarding adverse effects (especially adverse long-term effects) and insufficient information regarding the vaccine itself.

Improving the acceptance rates of COVID-19 vaccines will require a collaborative effort among a variety of experts. This collaborative effort should be aimed specifically at reducing hesitancy and raising awareness about vaccinations. This effort will likely need to include the government, public health decision makers, medical experts, and universities across the United States. Much is known about how to increase uptake in those who want to be vaccinated. Less is known about how to increase uptake in those without an intent to get vaccinated or how to increase uptake by modifying attitudes and beliefs. Some findings do suggest that confidence in physicians and public health officials can be instrumental in allaying people's fears and that this is a way to improve vaccination rates. Some studies suggest that those who are hesitant will reconsider as more information becomes available, but it is difficult to determine how much and what type of information may be necessary to convince unvaccinated individuals. Messages about vaccine safety and the ability to return to life as normal have shown to be the most effective types of messages at increasing actual vaccinations [2]. Developing and disseminating vaccination messaging, along with correcting misinformation, will continue to be important as nearly one-third of Americans still report a wish to take a wait-and-see approach, and 20\% remain quite reluctant [15]. There is work to be done. Estimates vary, but public health experts suggest $80-85 \%$ of Americans would need to be vaccinated for the country to achieve herd immunity. Many counties in Indiana are still at less than half of their eligible populations being vaccinated. Some counties are lower than $32 \%$ of those eligible fully vaccinated and one county with less than $34 \%$ of people receiving at least one dose [4].

\section{Methods}

This study was conducted at a mid-sized public university in Indiana. Students were distributed in two online surveys sent out one week apart. Survey questions included demographic information, intention to receive the vaccination, and reasons for hesitance when indicated.

\section{Data Analysis}

Consisted of summary statistics (frequencies and percentages) and ordinal logistic regression analysis. We performed an ordinal logistic regression analysis to determine the contribution of various predictor variables on indicating having no intention to receive the COVID-19 vaccine. An affirmative response concerning the intention to receive the COVID-19 vaccine was entered as the reference group for the regression model. The predictor variables included sociodemographic variables as well as attitudes and behaviors related to COVID-19 and the COVID-19 vaccine. Sociodemographic factors included gender, age, race, class status, and first generation status in terms of college attendance. Attitudinal variables included concerns with potential vaccine's side effects, lack of safety, lack of efficacy, needles, expense, location, as well as distrust of the vaccine efficacy, medical research, and government agencies. Health and behavioral factors related to COVID-19 included one's previous diagnosis of COVID-19, family members' diagnosis of COVID-19, and family member's receipt of the COVID-19 vaccination. IBM SPSS Version 27 was used to perform logistic regression analyses.

\section{Results}

A total of 1,653 students enrolled at a public Midwestern university completed the survey. Participants were described primarily as undergraduate students (74\%), female (61\%), and White $(81 \%)$. Although $50 \%$ of students had been vaccinated prior to data collection, $50 \%$ had not been. Among the 793 students who had not been vaccinated, $49 \%$ had no intention of getting vaccinated, and $22 \%$ were undecided. Interestingly, none of the students who indicated they intended to receive the vaccine provided responses to the most of the survey items. Therefore, we did not include these students in the summary statistics or Chi-square analyses. Thus, only students who indicated they did not intend to receive the COVID-19 vaccine and those who were undecided concerning their intention to receive the vaccine were included in the analysis. 
Using cross-tabulations with Chi-square analysis, we determined the breakdown of students' intentions of not receiving the COVID-19 vaccine or being undecided concerning their intentions by the demographic factors of age, gender, race, class status, and being a first generation college student. In terms of age, the group of students between the ages 24 and 29 had the highest percentage (79.3\%) reporting they did not intend to receive the COVID-19 vaccine. For the remainder of the examined demographic variables (including group sizes with 10 or more participants), the highest percentages of students who did not intend to receive the COVID-19 vaccine were graduates (49.1\%), multiracial/ other race $(73.7 \%)$, and those who were not First Generation (70.1\%). However, only gender was significantly associated with vaccine refusal, as males $(74.3 \%)$ were significantly more likely than females $(66.2 \%)$ to indicate having no intention to receive the COVID-19 vaccine, $X^{2}(1, N=554)=4.22$, $p=0.040$.

Participants were presented with potential barriers that may prevent them from receiving the COVID-19 vaccine. Primary reasons for hesitancy included not trusting the vaccine was fully tested (85.0\%), fear of potential side effects (78.0\%), not trusting the vaccine is safe $(72.0 \%)$, not trusting the US government (61.0\%), and having read negative reports from the media about the vaccine $(60.0 \%)$. The least frequently cited reasons for vaccine hesitancy and refusal were the expense of the vaccine $(1.0 \%)$ and being advised by a medical provider not to get the vaccine $(8.0 \%)$. Percentages are presented in Fig. 1.

Next, we used cross-tabulations with Chi-square analyses to determine if there were an association between a perception or behavior and refusal or hesitancy to receive the COVID-19 vaccine. Students who reported family members had not received the COVID-19 vaccine, $X^{2}(1, N=560)=8.49, p=0.004$; they did not trust the $\mathrm{U}$. S. government, $X^{2}(1, N=510)=4.22, p=0.040 ;$ did not trust health care providers, $X^{2}(1, N=509)=4.85, p=0.028$; they did not trust the vaccine had been fully tested, $X^{2}(1, N=509)=7.93, p=0.005$; they believed that the vaccine was not safe, $X^{2}(1, N=509)=27.78, p<0.001$; they believed that the vaccine could not protect them from getting COVID-19, $X^{2}(1, N=507)=27.95, p<0.001$; and they indicated that religious beliefs prevented them from receiving the vaccine, $X^{2}(1, N=507)=9.82, p=0.002$ were more likely to report they had no intention of receiving the COVID-19 vaccine (Table 1). However, students who reported they did

\section{Summary of Reported Factors That May Increase Vaccine Hesitancy $(n=561)$}

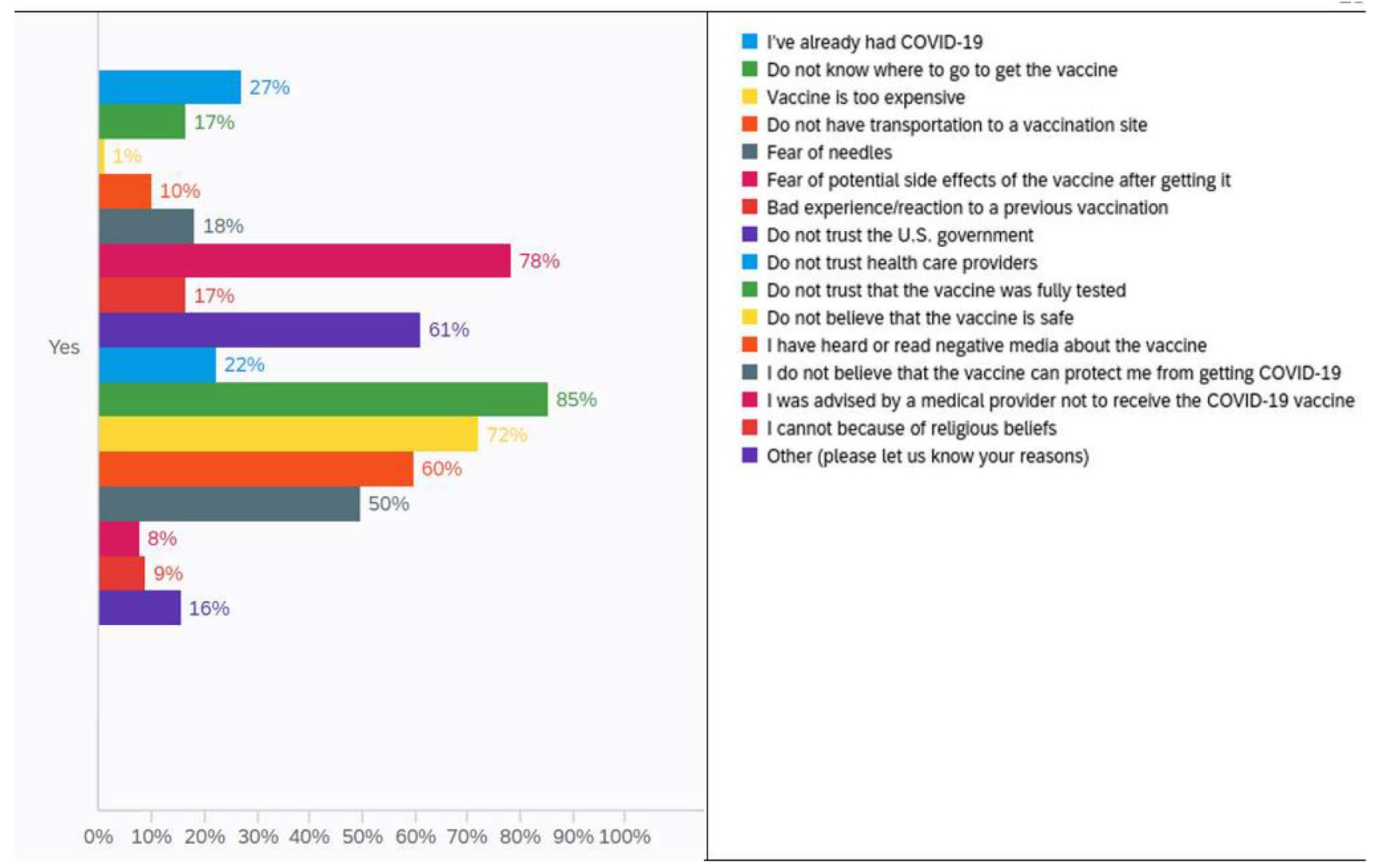

Fig. 1 Summary of reported factors that may increase vaccine hesitancy $(n=561)$ 
Table 1 Cross-tabulations of intention to receive the COVID-19 vaccine by perception and behaviors among students at a Midwestern State University $(\mathrm{n}=561)$

\begin{tabular}{|c|c|c|c|c|c|c|}
\hline \multirow[t]{2}{*}{ Intention to receive COVID-19 vaccine } & \multicolumn{2}{|l|}{$\begin{array}{l}\text { Does not intend to } \\
\text { receive COVID-19 } \\
\text { vaccine }\end{array}$} & \multicolumn{4}{|l|}{$\begin{array}{l}\text { Undecided concerning inten- } \\
\text { tion to receive COVID-19 } \\
\text { vaccine }\end{array}$} \\
\hline & $n$ & $\%$ & $n$ & $\%$ & $X^{2}$ & $p$ \\
\hline \multicolumn{7}{|l|}{ Fear of potential side effects $(N=512)$} \\
\hline Yes & 265 & 64.6 & 145 & 35.4 & 17.87 & $<0.001$ \\
\hline No & 88 & 86.3 & 14 & 13.7 & & \\
\hline \multicolumn{7}{|l|}{ Do not trust US government $(N=510)$} \\
\hline Yes & 240 & 75.0 & 80 & 25.0 & 16.20 & $<0.001$ \\
\hline No & 110 & 57.9 & 80 & 42.1 & & \\
\hline \multicolumn{7}{|c|}{ Do not trust the vaccine was fully tested $(N=509)$} \\
\hline Yes & 317 & 70.9 & 130 & 29.1 & 7.93 & 0.005 \\
\hline No & 33 & 53.2 & 29 & 46.8 & & \\
\hline \multicolumn{7}{|l|}{ Belief that vaccine is not safe $(N=510)$} \\
\hline Yes & 285 & 75.4 & 93 & 24.6 & 27.78 & $<0.001$ \\
\hline No & 67 & 50.8 & 65 & 49.2 & & \\
\hline \multicolumn{7}{|c|}{$\begin{array}{l}\text { Belief that vaccine cannot protect me from getting } \\
\text { COVID-19 }(N=507)\end{array}$} \\
\hline Yes & 207 & 79.6 & 53 & 20.4 & 27.95 & $<.001$ \\
\hline No & 143 & 57.9 & 104 & 42.1 & & \\
\hline \multicolumn{7}{|l|}{ Do not trust health care providers $(N=509)$} \\
\hline Yes & 89 & 76.7 & 27 & 23.0 & 4.85 & 0.028 \\
\hline No & 259 & 65.9 & 134 & 34.1 & & \\
\hline \multicolumn{7}{|c|}{ Family member received COVID-19 vaccine $(N=560)$} \\
\hline Yes & 218 & 64.5 & 120 & 35.5 & 8.49 & 0.004 \\
\hline No & 169 & 76.1 & 53 & 23.9 & & \\
\hline \multicolumn{7}{|c|}{ No vaccine because of religious beliefs $(N=511)$} \\
\hline Yes & 41 & 89.1 & 5 & 10.9 & 9.82 & 0.002 \\
\hline No & 310 & 66.7 & 155 & 33.3 & & \\
\hline
\end{tabular}

not have a fear of side effects from the vaccine were more likely to report they did not intend to receive the vaccine, $X^{2}(1, N=512)=17.87, p<0.001$.

\section{Discussion}

The results of the current study confirmed a number of findings from previous research regarding vaccine hesitancy. More than half of the students in the current study reported they either intended to receive the COVID-19 vaccine or were undecided about receiving it, which is consistent with Synott's findings [15]. CDC household panel surveys of a US representative sample of adults ages 18-64 conducted between September and December 2020 revealed younger adults, women, non-Hispanic Blacks, and adults with lower levels of education were more likely to identify as notintending to receive the vaccination [13]. Ideally, targeted interventions can be developed for groups identified as uninterested in pursuing vaccination.
In this study, concerns regarding vaccine safety and efficacy significantly predicted student's intentions to avoid the vaccine. Students in the current study share similar concerns expressed in the Fridman, Gershon, and Gneezy study [7] regarding the safety of the vaccine. A review of over 100 US and international surveys related to acceptance of the COVID vaccination revealed similar findings: concerns regarding the safety of the vaccination, fear of side effects and uncertainty of the overall effectiveness of the vaccine [12]. A survey of 1062 South Carolina college students' attitudes towards COVID-19 identified concerns with potential side effects and long-term harm as primary contributing factors towards vaccine hesitancy [14]. At the time of data collection, none of the COVID-19 vaccines had received FDA approval. It is unknown as to whether or not additional time, credible information, and FDA approval would have influenced vaccine hesitancy among this particular population.

Concerns regarding vaccine hesitancy have been compared in men and women. In general, men were more receptive of the COVID vaccine compared to women, and women 
tended to be more concerned with the potential side effects of the vaccine $[6,12]$. A study regarding vaccine hesitancy by Cerda and Garcia [6] found that the level of education was positively related to vaccine hesitancy. An Australian study of COVID-19 vaccine willingness found that women with bachelor's degrees were almost two times more likely to be uncertain about receiving the vaccine compared to men [1]. It is possible that there are additional factors specific to women that contribute to vaccine hesitancy.

In the current study, having family members who had not been vaccinated significantly contributed to participant's intentions to also refrain from receiving the vaccine. It is possible that in these situations, additional family members also expressed a general lack of distrust of the government and perhaps doubted the safety and efficacy of the vaccine which is consistent with previously reported US survey results. This particular study did not include political party affiliation; however, a review of several US COVID-related surveys that included questions pertaining to political party affiliation showed an overall higher acceptance rate for participants who categorized themselves as Democrats [12].

Students in this study expressed a relatively common concern that they "have heard or read negative media" about the vaccine. Social media platforms can provide a means for proliferation of conspiracy theories and politicized views regarding the vaccine, as well as unmoderated content. For example, textual analysis of content posted to a newer social media platform known as Parler identified major themes such as government and medical distrust and the theory that the COVID-19 vaccine is a form of population control [3]. While social media use was not specifically included in the parameters of this study, the extent of social media use as well as the platforms used may affect the credibility of vaccine information obtained.

\section{Conclusion}

College students are a population that may be more hesitant to receive the vaccination than others. Several factors may be influencing this including education, attitudes, and where they receive their information. Targeted messaging may be a way to influence those who are hesitant to receive the COVID-19 vaccination.

Authors' Contribution All authors contributed to survey creation, data collection, data analysis, and writing.

Funding The authors received no grant funding from any agency in the public, commercial, or not-for-profit sectors.

Data Availability Authors have data saved in SPSS.
Code Availability Not applicable.

\section{Declarations}

Conflict of interest We have no relationships or interests that influenced or biased this work.

Ethical Approval Not applicable.

Consent to Participate All participants gave consent to participate via informed consent.

Consent for Publication The authors give the journal consent to publish.

\section{References}

1. Alley, S. J., Stanton, R., Browne, M., To, Q. G., Khalesi, S., Williams, S. L., Thwaite, T. L., Fenning, A. S., \& Vandelanotte, C. (2021). As the pandemic progresses, how does willingness to vaccinate against COVID-19 evolve? International Journal of Environmental Research and Public Health, 18(2), 797. https:// doi.org/10.3390/ijerph18020797

2. Ashworth, M., Thunstrom, L., Cherry, T. L., \& Newbold, S. C. (2021). Emphasize personal health benefits to boost COVID-19 vaccination rates. Proceedings of the National Academy of Sciences, 118(32), 1-14.

3. Baines, A., Ittefaq, M., \& Abomewao, M. (2021). \#Scamdemic, \#Plandemic, or \#Scaredemic: What Parler social media platform tells us about COVID-19 pandemic. Vaccines, 9(5), 421. https:// doi.org/10.3390/vaccines 9050421

4. Centers for Disease Control and Prevention. (2021, September). COVID data tracker. U.S.

5. Department of Health and Human Services, National Institutes of Health. https://covid.cdc.gov/covid-data-tracker/\#datatracker-h.

6. Cerda, A. A., \& Garcia, L. Y. (2021). Hesitation and refusal factors in individuals' decision making processes regarding a Coronavirus Disease 2019 vaccination. Frontiers in Public Health. https://doi.org/10.3389/fpubh.2021.626852

7. Cutler, D. M., \& Summers, L. H. (2020). The COVID-19 pandemic and the \$16trillion virus. Journal of the American Medical Association, 324, 1495-1496.

8. Fridman, A., Gershon, R., \& Gneezy, A. (2021). COVID-19 and vaccine hesitancy: A longitudinal study. PLOS ONE, 16(4), e0250123. https://doi.org/10.1371/journal.pone.0250123

9. Harrison, E. A., \& Wu, J. W. (2020). Vaccine confidence in the time of COVID-19. European Journal of Epidemiology, 35, $325-330$.

10. Iboi, E. A., Ngonghala, A. B., \& Gumel, A. B. (2020). Will an imperfect vaccine curtail the COVID-19 pandemic in the US? Infectious Disease Modelling, 5, 510-524.

11. Indiana Department of Health. (2021, September). Indiana COVID-19 Vaccination Dashboard. https://www.coronavirus.in. gov/2393.htm

12. Lin, C., Tu, P., \& Beitsch, L. M. (2021). Confidence and receptivity for COVID-19 vaccines: A rapid systematic review. Vaccines, 9(16), 1-41. https://doi.org/10.3390/vaccines9010016

13. Nguyen, K. H., Srivastav, A., Razzaghi, H., Williams, W., Lindley, M. C., Jorgensen, C., Abad, N., \& Singleton, J. A. (2021). COVID-19 vaccination intent, perceptions, and reasons for not vaccinating among groups prioritized for early vaccination-United States, September and December 2020. American Journal of 
Transplantation, 21(4), 1650-1656. https://doi.org/10.1111/ajt. 16560

14. Qiao, S., Tam, C. C., \& Li, X. (2021). Risk exposures, risk perceptions, negative attitudes toward general vaccination, and COVID19 vaccine acceptance among college students in South Carolina. American Journal of Health Promotion. https://doi.org/10.1177/ 08901171211028407

15. Rosenbaum, L. (2021). Escaping Catch-22 - Overcoming COVID vaccine hesitancy. The New England Journal of Medicine, 384(14), 1367-1371. https://doi.org/10.1056/NEJMms2101220

16. Saied, S. M., Saied, E. M., Kabbash, I. A., \& Abdo, S. A. E. F. (2021). Vaccine hesitancy: Beliefs and barriers associated with COVID-19 vaccination among Egyptian medical students. Journal of medical virology, 93(7), 4280-4291. https://doi.org/ 10.1002/jmv.26910 Sallam, M. (2021). COVID-19 Vaccine
Hesitancy Worldwide: A concise systematic review of vaccine acceptance rates. Vaccines, 9(2), 160. https://doi:https://doi.org/ 10.3390 /vaccines 9020160

17. Synnott, K. (2021). Managing College Students' COVID-19 Vaccine Hesitancy. SSRN

18. Vergara, R. J. D., Sarmiento, P. J. D., \& Lagman, J. D. N. (2021). Building public trust: A response to COVID-19 vaccine hesitancy predicament. Journal of Public Health, 43(2), e291-e292.

Publisher's Note Springer Nature remains neutral with regard to jurisdictional claims in published maps and institutional affiliations. 